\title{
Aides à l'apprentissage et retour pédagogique dans les sites d'apprentissage des langues en ligne
}

\section{Christian Ollivier et Gerda Weiss}

\section{OpenEdition}

Édition électronique

URL : http://journals.openedition.org/asp/1238

DOI : 10.4000/asp. 1238

ISBN : 978-2-8218-0394-7

ISSN : 2108-6354

Éditeur

Groupe d'étude et de recherche en anglais de spécialité

Édition imprimée

Date de publication : 1 novembre 2003

Pagination : 143-154

ISSN : 1246-8185

Référence électronique

Christian Ollivier et Gerda Weiss, « Aides à l'apprentissage et retour pédagogique dans les sites d'apprentissage des langues en ligne », ASp [En ligne], 41-42 | 2003, mis en ligne le 29 avril 2010 consulté le 19 avril 2019. URL : http://journals.openedition.org/asp/1238; DOI : 10.4000/asp.1238

Ce document a été généré automatiquement le 19 avril 2019

Tous droits réservés 


\title{
Aides à l'apprentissage et retour pédagogique dans les sites d'apprentissage des langues en ligne
}

\author{
Christian Ollivier et Gerda Weiss
}

1 Voici un beau site d'apprentissage d'une langue, haut en couleurs et même en animations qui incitent à cliquer pour découvrir les contenus. La première page donne envie d'apprendre. La désillusion guette cependant au premier clic. Les activités proposées ne correspondent pas vraiment à vos attentes ni à votre niveau d'apprenant et rien ne l'indiquait. A l'intérieur des activités : aucune aide en vue et, pour finir, le système vous indique, sans autre forme de procès, un score désastreux avec peut-être en prime les réponses qu'il aurait fallu donner et que vous ne comprenez pas. Certes, ce tableau ne représente pas la réalité de tous les sites d'apprentissage des langues sur Internet, mais il ne s'agit pas non plus d'une mauvaise fiction. C'est à partir du constat de nombreux déficits, mais aussi sur la base des recherches existantes en didactique de l'enseignement - apprentissage des langues sur Internet et d'expériences antérieures ${ }^{1}$ que le Centre de langues de l'Université de Salzburg s'est lancé dans la conception et la réalisation de NetHorizon, un site d'apprentissage du français langue étrangère pour lycéens et étudiants autrichiens.

2 Dans le présent article, nous reprendrons les éléments de réflexion théorique qui constituent la base de notre projet pour ensuite montrer comment ceux-ci peuvent être mis en pratique. Nous nous concentrerons sur deux points essentiels qui constituent la majeure partie du dialogue entre utilisateur et système : les aides à l'apprentissage et les retours pédagogiques qu'un tel site, que nous avons conçu pour être utilisable en autonomie, doit offrir à ses utilisateurs pour qu'un apprentissage efficace soit possible. 


\section{Autonomie, construction des projets et parcours d'apprentissage}

3 Proposer des activités d'apprentissage interactives revient, dans notre cas, à proposer des activités réalisables en autonomie et donc à remettre le terme même d'autonomie au centre de nos préoccupations. Car si, depuis plusieurs décennies déjà, l'autonomie de l'apprenant ressort comme une des notions centrales dans le domaine de l'apprentissage des langues ${ }^{2}$, on constate un fossé important entre pratique et théorie. Cette dernière reste trop souvent encore lettre morte ou déclaration de principe, notamment sur les sites d'apprentissage qui, de par leur facile accessibilité à un large public, la liberté de navigation offerte à l'utilisateur et les possibilités techniques offertes, pourraient pourtant, ou devraient, favoriser la prise d'autonomie. Nous rappellerons donc ci-dessous la conception de l'autonomie que nous avons retenue pour développer ensuite les possibles implications pratiques.

4 Vouloir favoriser l'autonomie implique de renoncer à une vision instructionniste d'un processus d'enseignement - apprentissage centré sur un enseignant qui prendrait toutes les décisions (définition des objectifs, moyens, modalités temporelles et spatiales de l'apprentissage...) et de (re)donner d'importantes libertés aux apprenants, de leur laisser prendre des décisions sur leur apprentissage - Philippe Morin parle même de «prise de contrôle» (2000: 31). Henri Portine définit l'autonomie comme la faculté de «savoir se fixer des objectifs que l'on peut atteindre et gérer son temps et ses activités en fonction de ces objectifs au sein d'un ensemble plus grand qui détermine ce qui est possible et ce qui ne l'est pas » (1998: 76). Autrement dit: «l'autonomie, c'est construire un projet d'action et gérer la réalisation de ce projet au sein d'une structure qui définit les contraintes globales et apporte une aide lorsqu'elle est nécessaire" (1998: 73). L'autonomie, ce n'est donc en aucun cas « se débrouiller tout seul ». L'autonomie s'inscrit dans un ensemble structuré qui va soutenir et éventuellement guider l'apprenant dans son projet. Proposer des activités réalisables en autonomie ne signifie donc pas, comme certains concepteurs de sites semblent le penser, laisser l'apprenant livré à lui-même sous prétexte que c'est à lui de construire son apprentissage et de prendre les décisions. Il convient en effet de garder présent à l'esprit que l'autonomie n'est pas donnée et qu'elle nécessite un apprentissage de la part du sujet apprenant : « elle est à construire et non à décréter ", rappellent Maguy Pothier, Anne Iotz et Christine Rodrigues (2000: 150). Elle s'acquiert dans un processus comprenant reculs et avancées (Cf. Portine 1998: 74), comme le faisait déjà remarquer Vygotski pour tout apprentissage. Il importe donc que l'apprenant soit accompagné et soutenu dans son parcours d'apprentissage.

5 Le rôle de l'accompagnement par un tuteur a largement été discuté par la recherche (cf. entre autres Duda 1984, Holec 1985, Abé \& Gremmo 1985, Demaizière \& Foucher 1998). Nous ne pouvons que souligner, à notre tour, l'importance de la présence d'une personne experte, consultable par l'apprenant, afin de mieux gérer son parcours d'apprentissage. Mais nous ne développerons pas ce point dans le cadre d'un article dont ce n'est pas le propos et nous concentrerons ci-dessous sur l'accompagnement et l'aide à l'apprentissage qui peut être apportée à l'apprenant par l'intermédiaire du système informatique.

6 Afin de mieux montrer parmi les différents types d'assistance envisageables ceux que nous avons retenus et d'expliquer ces choix, nous reviendrons rapidement sur l'analyse 
de l'autonomie en formation ouverte et à distance que fait le collectif de Chasseneuil au terme de la Conférence de consensus :

Les dimensions principales de l'autonomie sont la motivation (sens, projet, finalités, enjeux personnels) et les compétences de niveau « méta ", c'est-à-dire les capacités métacognitives (apprendre à apprendre, identifier et gérer des ressources, [...] maitriser les techniques et outils d'apprentissage, etc.). $(2000: 6)$

7 Si l'on retient cette définition, un site d'apprentissage doit comporter des éléments visant, d'une part, à renforcer la motivation et, d'autre part, à développer les nécessaires capacités métacognitives. Il importe donc, dans un premier temps, de proposer à l'apprenant des points de repère clairs lui permettant de définir ou redéfinir ses objectifs et de choisir les activités qui l'aideront à atteindre ces objectifs. Il est primordial que les utilisateurs soient assistés dans leur choix des activités : ils doivent pouvoir repérer rapidement et aisément ce que chaque activité peut leur permettre d'apprendre. Non seulement la présentation des objectifs va aider l'apprenant à s'orienter et à trouver les ressources qui lui conviennent, mais elle peut également attirer son attention sur d'éventuels objectifs auxquels il n'avait pas pensé. L'utilisateur peut ainsi préciser, voire repenser, son projet d'apprentissage et intégrer dans son projet d'apprentissage des objectifs qu'il n'avait pas envisagés initialement. En cela, le système joue un rôle de tuteur d'apprentissage proche de celui fixé, par exemple, dans les expériences du CRAPEL ${ }^{3}$.

8 Si les concepteurs de sites pédagogiques ne peuvent guère avoir d'influence sur les autres éléments plus intrinsèques de la motivation (enjeux personnels, sens, finalités), ils peuvent cependant contribuer à augmenter la motivation de l'apprenant en lui proposant des activités variées comportant des éléments inattendus qui permettront de fixer son attention et son intérêt. Nous veillons ainsi à proposer en fin d'activité un feedback final varié à caractère de surprise (fanfares, animations, images...) qui doit représenter en soi une motivation supplémentaire pour l'apprenant qui réalisera aussi l'activité pour découvrir le retour final (Cf. Keller 2000). 
Figure 1. Page d'accueil de l'unité 1

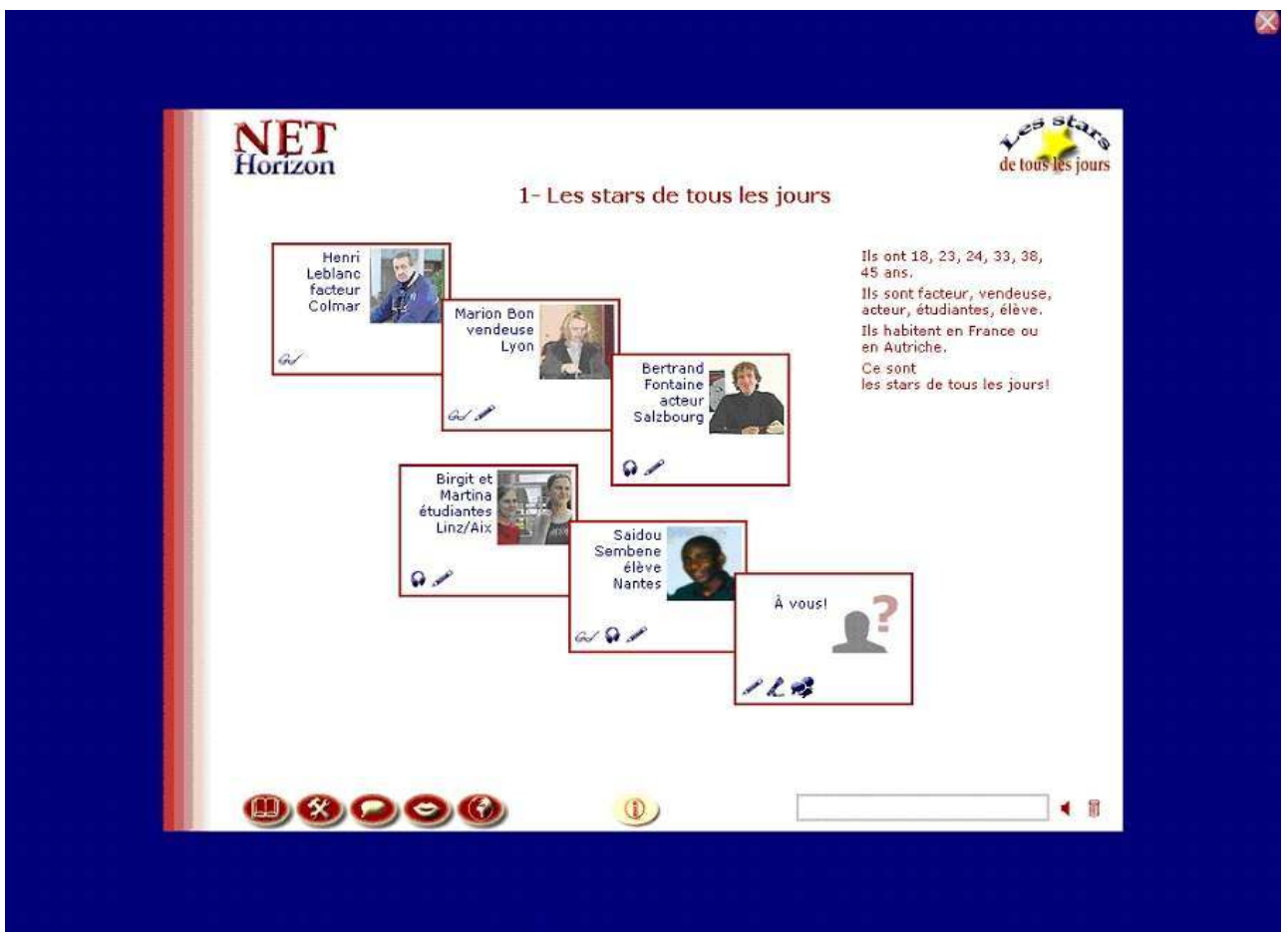

Pour ce qui est du deuxième aspect, les capacités métacognitives, nous attachons une importance toute particulière à la mise au point d'un système de navigation aussi clair et souple que possible permettant à l'utilisateur une orientation intuitive, adaptée aux besoins de l'apprentissage et permettant à chaque instant d'interrompre une séquence pour revenir aux pages de choix. Ainsi, sur le site en construction de Net-Horizon, un logo placé en haut à gauche de chaque écran permet un retour immédiat au tout début du site (page de choix des unités) tandis qu'un logo, placé en haut à droite, permet un retour au départ de l'unité où l'apprenant trouve une page de choix des activités incluant la description des objectifs poursuivis. De plus, une barre de navigation, présente en bas de chaque page, donne un accès direct aux ressources grammaticales, lexicales, phonétiques et (inter-)culturelles liées directement aux objectifs communicatifs des activités. Le va-etvient aisé entre ces ressources et les activités plus communicatives de l'unité est assuré par le système. Ainsi, un utilisateur décidant d'avoir recours, par exemple, à un complément grammatical pour mener à bien une activité peut se diriger vers celui-ci et revenir ensuite d'un seul clic vers l'activité sur laquelle il travaillait (cf. infra).

10 Afin de clarifier la navigation iconique, une page d'explication des symboles utilisés, rédigée en allemand, langue maternelle du groupe cible, et en français, est accessible à partir de la première page de l'unité. Il est en effet essentiel - et pourtant plusieurs sites semblent n'accorder qu'une très faible importance à cet aspect - que l'utilisation du système et son éventuel décodage représente une charge cognitive aussi peu importante que possible. Une grande souplesse du système et une importante simplicité d'utilisation sont impératives sous peine de démotiver l'apprenant (qui n'est pas toujours habitué à l'apprentissage avec un ordinateur) et donc d'entraver l'apprentissage.

11 On le voit clairement, impératifs ergonomiques, techniques et choix didactiques se rejoignent et sont interdépendants. Cela ressort également clairement dans la conception des unités apprentissage où il importe de trouver un équilibre entre liberté de décision de 
l'apprenant et guidages. Dans le cas de Net-Horizon, une première page de chaque unité, donnera accès à de micro séquences créées autour de micro objectifs de communication. $\mathrm{Au}$ sein de ces séquences, nous avons opté pour une navigation linéaire, puisque les activités suivent une progression. L'utilisateur aura cependant toujours la possibilité de sauter une étape, de revenir sur une activité précédente ou, comme nous le précisions cidessus, de revenir à la page de choix grâce aux logos placés en haut de page. Ainsi, il garde, s'il le souhaite, le contrôle complet de son cheminement. Nous lui laissons la liberté de construire son parcours, tout en lui proposant des cheminements qu'il empruntera ou non :

L'apprenant dirige sa propre expédition. Il décide de son cheminement. C'est à lui [...] que revient la décision d'aller de l'avant ou de revenir éventuellement en arrière de façon à explorer les chemins qu'il n'avait pas choisis précédemment. [...] Chaque apprenant construit, dans une certaine mesure, son propre scénario pédagogique. (Demaizière, Dubuission $1992: 74$ )

\section{Autonomie et aides à la réalisation des tâches}

Aider l'apprenant à construire son scénario pédagogique est essentiel, l'aider à effectuer les tâches d'apprentissage qui lui sont proposé l'est tout autant. Or, la présence d'aides accessibles directement depuis les pages d'activités est une pratique encore peu répandue sur Internet bien qu'il soit très démotivant pour l'apprenant de se retrouver face à une activité et de ne pas parvenir à la réaliser.

La première aide à apporter prend la forme de consignes claires, faciles à consulter et à comprendre et aussi succinctes que possible. Les concepteurs sont, en ce point, confrontés à des difficultés généralement inconnues des concepteurs de manuels ou de fiches d'exercice. Les habitudes de lecture sur papier font que généralement l'apprenant lit ou du moins survole la consigne qui précède l'exercice avant de se lancer dans celui-ci. Ceci n'est pas forcément le cas sur une page web où la lecture ne se fait pas de gauche à droite et de haut en bas. Pour remédier à cette difficulté, nous ressentons le besoin de proposer des activités dont on devine intuitivement la tâche à accomplir, les consignes proposées en allemand et en français au début - ne doivent servir qu'à renforcer la première impression.

14 Afin d'éviter toute frustration devant une activité que l'apprenant ne réussirait pas à réaliser, nous suivons entièrement l'argumentation de J. Rézeau (2001) et plaidons en faveur d'un accès aisé à la solution. Il ne s'agit en aucun cas d'encourager la paresse - la responsabilité de l'apprentissage revient toujours à l'apprenant; quelle que soit la situation (enseignement présentiel ou phase d'auto-apprentissage), c'est en dernière instance lui qui décide s'il veut ou non apprendre -, nous entendons seulement donner à l'apprenant la possibilité de voir la solution ; à lui de choisir s'il a ou non recours à cette aide. Nous sommes convaincus que la solution peut aider l'apprenant à comprendre ce que l'activité qu'il est en train de réaliser doit lui permettre d'apprendre. Nous avons cependant pu constater lors d'expérimentations que les apprenants avaient relativement peu recours à ce qu'ils ressentent parfois comme une solution de facilité; tout comme J. Rézeau (2001), nous notons chez beaucoup d'apprenants un "acharnement " certain à réussir par eux-mêmes. Ceci est peut-être dû à des modes d'apprentissage personnels ou à une conception traditionnelle de l'apprentissage qui, trop souvent, fait de chaque exercice un test pour l'apprenant. Or, on peut utiliser les possibilités des nouvelles technologies pour proposer de nombreuses aides et faire en sorte que les exercices et 
activités soient vraiment ressentis comme tels. C'est la raison pour laquelle nous avons renoncé à des indications chiffrées indiquant un taux de réussite ou d'échec (cf. cidessous). Nous préférons aider l'apprenant en lui proposant des pistes lui permettant de réaliser les activités.

Chaque fois que cela nous semble opportun, voire nécessaire, nous incluons donc des aides permettant d'effectuer avec succès les activités proposées. Nous distinguons deux types d'aide: l'aide contextualisée devant faciliter la résolution d'une micro tâche et l'aide globale se rapportant à l'ensemble de l'activité.

L'aide contextualisée, que nous avons souvent décomposée en plusieurs étapes, offre des informations choisies pour permettre à l'apprenant de trouver une réponse ponctuelle. Qu'il nous soit permis de fournir un exemple pour clarifier notre point de vue. Dans une activité comprenant un texte lacunaire, l'apprenant trouvera à côté de chaque item un bouton qui lui permettra d'avoir accès à une première aide. Ce pourra être, par exemple, une sorte d'aide-mémoire proposant des indices ou réactivant des associations créées auparavant, ou bien un rappel de l'élément précis d'une règle de grammaire en rapport direct avec la micro tâche à réaliser. Dans de nombreux cas, un petit bouton supplémentaire donnant accès à une aide plus importante (autre indice ou réponse exacte par exemple) apparaîtra à côté de l'indice. L'apprenant garde ainsi le contrôle de l'ampleur de l'aide qu'il entend solliciter du système.

17 L'aide globale se rapporte à l'objectif général de l'activité dans son ensemble. Il pourra s'agir de pages reprenant diverses réalisations des intentions communicatives abordées ou de pages de découverte et de présentation de la grammaire, du lexique, etc. Nous pensons qu'il est essentiel que ces informations soient accessibles depuis l'activité et que le retour à l'activité soit aussi aisé que possible pour éviter que l'utilisateur ne se perde dans le système et qu'une partie de ses réponses soit effacée par des opérations de navigation. C'est la raison pour laquelle nous avons veillé à ce que l'apprenant puisse toujours revenir à son point de départ d'un seul clic et qu'il retrouve l'activité dans l'état d'avancement dans lequel il l'avait quittée.

On notera que toutes les aides sont disponibles, mais jamais imposées. Cela nécessitera pour certains un temps d'adaptation et parfois même une nouvelle façon de penser l'apprentissage, mais nous sommes persuadés que ces aides représentent un atout considérable par rapport au support papier beaucoup moins souple et configurable par son utilisateur. En n'imposant pas l'aide, nous tenons compte des apprenants qui n'en auront pas besoin ou qui ne souhaiteront pas y avoir recours. Nous entendons ainsi prendre en considération plusieurs façons d'apprendre différentes sans en privilégier une plus qu'une autre et surtout sans en condamner aucune. On retrouvera d'ailleurs ce souci de ne pas sanctionner dans la conception et la mise en œuvre des retours pédagogiques au sein du site en construction.

\section{Autonomie et retours pédagogiques ${ }^{4}$}

19 L'idée de retour pédagogique est liée à l'idée d'interaction entre deux partenaires, c'est-àdire à un processus d'échange d'information. Dans le dialogue interactif, le retour pédagogique est à percevoir comme réaction différenciée du système programmé pour analyser et répondre à une action de l'apprenant $t^{5}$. La façon dont cette analyse est structurée reflète les principes didactiques et pédagogiques selon lesquels le concepteur a créé ses activités 
d'apprentissage. Car c'est bien le concepteur qui doit, par anticipation, prévoir les actions de l'apprenant et définir les réactions du système.

Un grand nombre de didacticiels visent surtout à mesurer la performance de l'apprenant au détriment de la construction de savoir et du développement de l'autonomie. Dans beaucoup de cas, les activités sont élaborées d'une manière simple et répétitive et le retour, plus mécanique que pédagogique, fournit soit des scores, soit ce qu'on peut considérer comme une information minimale sur le statut de la réponse (correcte ou incorrecte). Il semble évident que de telles méthodes mènent l'apprenant automatiquement à éviter et à négliger l'erreur ou finissent par le démotiver si ses erreurs se multiplient. Étant ainsi réduit à un simple rôle de sanction ou de renforcement, le retour suit des principes qui ont leur origine dans la théorie behaviouriste et qui excluent les connaissances actuelles en matière de métacognition. Du point de vue d'une didactique moderne du multimédia favorisant l'apprentissage autonome (cf. supra), cette forme de retour ne peut qu'être qualifiée d'insatisfaisante.

21 Notre conception du retour pédagogique s'intègre dans une approche cognitive de l'apprentissage des langues qui perçoit l'erreur en tant qu'élément non seulement inévitable, mais aussi nécessaire dans le processus d'apprentissage. (Cf., entre autres, le modèle de traitement d'information de McLaughlin et al. (1983) ou le modèle ACT de Anderson (1985)). Par conséquent, nous suivons entièrement J. Rézeau qui constate que "le rôle du feedback n'est pas seulement de fournir un renforcement, mais des renseignements devant permettre à l'apprenant de prendre en charge la modification de sa production langagière.» (2001) Au lieu d'ignorer ou de sanctionner l'erreur, il nous paraît important de l'intégrer dans un retour pédagogique qui tienne compte - autant que faire se peut - de la réponse de l'apprenant, afin de permettre à celui-ci d'utiliser ses erreurs en tant que source d'information et possibilité de réflexion sur son processus d'apprentissage. De cette façon, le retour pédagogique joue un rôle essentiel sur le plan du développement des capacités d'autoévaluation et de gestion de l'apprentissage, telles que définies entre autres par Chamot et O'Malley (1994 : 375).

Le retour pédagogique en tant que source d'information informe l'apprenant de la façon la plus précise possible sur, premièrement, les conséquences de son action et, deuxièmement, sur l'état actuel de son savoir. Informer équivaut donc à fournir à l'apprenant une analyse de réponse ainsi que des repères pour son apprentissage. Le concepteur doit donc d'emblée se demander comment réaliser un retour pédagogique qui soit à la fois adapté à l'apprenant et efficace. Pour ce faire, il faut, d'une part, prendre en compte les contenus d'apprentissage et, par conséquent, le type d'activité et d'autre part, les caractéristiques de l'apprenant.

23 Les activités conçues pour des didacticiels multimédia proposent en général un choix de réponses assez limité. Cette restriction implique que les possibilités de réponse sont prévisibles par le concepteur et que le retour pédagogique est relativement facile à programmer. En contrepartie, vu que ce genre de feedback est forcément limité, il existe un risque non négligeable de programmer des retours extrêmement standardisés et monotones - le contraire précisément de ce que l'on veut obtenir. Il existe en outre des types d'activité qui proposent des questions plus ouvertes et invitent à donner des réponses plus libres et donc plus imprévisibles. Il va de soi que cela implique une analyse de réponse très complexe, ce qui rend la programmation très difficile, sinon impossible, dans la plupart des cas. Pour ce qui est des caractéristiques de l'apprenant, le concepteur 
doit en premier lieu faire un choix entre adapted feedback (retour pédagogique adapté) et adaptive feedback (retour pédagogique adaptatif). (Cf. Sales 1993 : 165-66)

Sales décrit l'adapted feedback en tant que stable dans le programme, c'est-à-dire que chaque apprenant reçoit, pour une même réponse, le même retour pédagogique lorsqu'il parcourt une unité. Le concept d'adapted feedback s'oppose au concept d'adaptive feedback qui constitue un retour pédagogique dynamique. Cela signifie que le programme est conçu de telle façon que chaque apprenant reçoit un retour pédagogique individualisé en fonction de ses performances précédentes. Un tel projet exige une analyse intensive des réponses possibles avant la création du programme ainsi qu'une programmation qui permette une analyse de réponse complexe pendant que l'apprenant parcourt les unités. La mise en pratique est donc liée à des coûts considérables. L'équipe du Centre de Langues de Salzbourg a agi selon ses capacités et a opté pour un retour pédagogique adapté, affiné par la prise en compte du groupe cible (public germanophone principalement étudiant et lycéen) et de l'environnement d'apprentissage particulier (enseignement en présentiel avec des phases d'apprentissage en autonomie tutorée ou non sur le site). Cette solution nous semble représenter un bon compromis entre adaptation et standardisation.

Afin d'ajuster le retour pédagogique à notre groupe cible nous avons, par exemple, recouru à la langue maternelle de l'apprenant lorsque les messages de retour nous semblaient être trop difficiles au niveau linguistique. Ainsi, le retour se présente soit en allemand, soit sous forme bilingue, soit uniquement en français. Dans ce dernier cas, nous utilisons systématiquement des mots et des structures simples ainsi qu'un soutien visuel pour assurer la compréhension.

$\mathrm{Au}$ cas où l'apprenant désirerait une évaluation sommative individualisée qui lui permette de mesurer ses progrès d'apprentissage, le site proposera des tests permettant à l'apprenant de chiffrer sa performance selon des critères prédéfinis. De plus, en fonction des résultats obtenus au test, le programme fournira également à l'apprenant des incitations à faire ou refaire certaines séquences afin de combler d'éventuelles lacunes. Ainsi, l'évaluation sommative sera directement reliée à une évaluation plus formative.

Une autre mesure d'individualisation a largement été développée ci-dessus: au lieu d'offrir un accès à la solution dans le cadre du retour pédagogique, nous avons opté pour des aides contextualisées auxquelles l'apprenant peut recourir ou non, selon ses besoins individuels.

Reste la question tout aussi fondamentale du moment propice au retour pédagogique. De nombreuses recherches à ce propos ont mené à des résultats et des positions controversées (cf. Kulhavy 1977, Dempsey \& Wager 1988). Nous avons opté pour un retour pédagogique qui répond directement et automatiquement à l'action de l'apprenant afin de l'informer sur la qualité de sa réponse mais aussi sur l'état du système. Autrement dit, il est nécessaire que l'utilisateur sache si le programme a réagi de quelque manière que ce soit à son action. L'utilisateur obtient donc une réaction immédiate pour chaque micro tâche et une réaction finale lorsqu'il a achevé l'activité avec succès. Il sait ainsi en permanence si ces actions sont correctes ou non et qu'aucun dysfonctionnement du système n'est intervenu. Ainsi, grâce à ces réactions constantes du système et au réseau d'aides proposées, l'apprenant est mis en situation de contrôle permanent de son apprentissage et incité à mettre en œuvre des stratégies métacognitives.

Si la perspective cognitive, que nous venons d'aborder, met l'accent sur la fonction informative du retour pédagogique, elle doit aussi la dépasser en se situant également au 
niveau affectif afin de stimuler également la motivation de l'apprenant sur son chemin vers l'autonomie. Selon le modèle ARCS de Keller (2000), l'attention représente un des éléments constitutifs de la motivation et peut être maintenue et renforcée grâce à quelques stratégies que le concepteur de sites d'apprentissage peut mettre en pratique en exploitant les nombreuses possibilités techniques du multimédia.

Nous retiendrons principalement la stratégie de la variability (cf. Keller 2000): afin d'éviter la monotonie et de maintenir l'attention, il semble opportun de varier autant que faire se peut le type de retour pédagogique proposé même si, comme le rappelle $\mathrm{J}$. Rézeau:

[...] on constate d'une part un enchaînement logique entre le type d'activité proposé à l'élève, le type d'analyse de réponse aboutissant à un type de feedback donné et d'autre part un taux d'efficacité directement lié à l'association d'un type d'activité avec le type de feedback le mieux approprié. (2001)

31 Nous proposons ci-dessous une classification des retours pédagogiques par degré de complexité selon les définitions de Ross et al. (1993 : 180-82) en précisant pour quels types d'activités ils nous semblent tout particulièrement appropriés.

\section{Knowledge of response, $\mathrm{KR}$}

Ce retour pédagogique indique si la réponse est correcte ou incorrecte. Nous avons utilisé ce type de feedback pour les activités de type vrai/faux, appariement et repérage. De telles activités ne proposant guère que deux options : l'une correcte, l'autre non, un feedback plus complexe risquerait de fournir un surplus d'information inutile. Dans certains cas cependant, on peut envisager de préciser pourquoi l'élément choisi n'est pas le bon.

\section{Knowledge of correct response, $\mathrm{KCR}$}

Ce type de feedback indique la solution correcte. Nous renonçons à ce type de retour et préférons offrir un accès à la solution à travers les aides. (cf. supra)

\section{Answer-until-correct feedback, AUC}

Ce type de retour pédagogique est une extension des types $K R$ et $K C R$. Il implique que l'apprenant soit informé, après avoir donné une réponse erronée, sur le nombre d'essais dont il dispose pour trouver la ou les solution(s) correcte(s). Suivant le principe de l'autonomie de l'apprenant, nous avons construit le retour pédagogique de telle manière que la possibilité d'un nouvel essai existe toujours.

\section{Elaborated feedback}

Le concept de l'élaboration se réfère à "any information given beyond the initial verification ». (Ross et al. 1993 : 182) Ce type de retour est sans doute le plus utile quand il s'agit de fournir à l'apprenant une analyse de réponse plus détaillée. Ceci concerne surtout les types d'activité qui proposent un choix de réponses moins restreint, telles que les activités lacunaires ou les QCM. Nous proposons, pour l'instant, trois variantes de retour élaboré.

Pour toutes les formes d'activité lacunaire, il existe une analyse d'erreur, permettant de conserver les éléments de réponses corrects (lettres, mots...) et de supprimer les éléments incorrects. De plus, un retour de type $K R$ accompagne cette analyse détaillée.

Outre ces analyses de réponses, nous proposons des informations supplémentaires soit en réaction à une réponse de l'apprenant soit par l'intermédiaire des aides contextualisées, comme nous les décrivions ci-dessus. Les activités de type QCM sont particulièrement propices à la mise en œuvre de ce type de feedback puisque réponses correctes et 
incorrectes sont connues des concepteurs qui les ont choisies par avance. Il est donc possible de proposer des informations précises en fonction des choix de l'apprenant, ces informations pourront soit aider à trouver la bonne solution soit apporter des compléments à une solution correcte.

Nous avons également travaillé sur la mise au point de retours communicatifs qui s'intègrent parfaitement, nous semble-t-il, aux activités ayant ce même caractère communicatif. Dans ce cas de figure, l'apprenant obtient pour toute action correcte une réaction telle qu'elle pourrait exister dans une interaction humaine. Ainsi, dans une activité permettant d'apprendre à demander à quelqu'un des informations sur sa personne, l'apprenant qui inscrira une question permettant de demander son nom à quelqu'un entendra une personne lui donner son nom.

Pour les raisons que nous énoncions ci-dessus et considérant avec Dorthy M. Hoska (1993: 116) qu'une atmosphère de compétition s'avère généralement nuisible à la construction du savoir, les retours pédagogiques ne proposent aucun score. Seules les activités à caractère ludiques (Memory par exemple) font exception, le score devant inciter à refaire le jeu pour obtenir un meilleur résultat.

Tous ces types de retours pédagogiques, liés souvent à des types d'activités, peuvent être variés en utilisant des canaux différents comme le prône John Keller (2000). Nous retenons, pour l'instant, trois modes de présentation des retours.

\section{- Le retour pédagogique visuel utilisant :}

- des images fixes : nous avons choisi des symboles (croix rouge, par exemple, pour une réponse inexacte) qui ont l'avantage de véhiculer le même contenu sémantique (indiquer si une réponse est correcte ou non) qu'un retour écrit tout en touchant l'utilisateur de façon plus directe.

- des images animées : l'animation est une des caractéristiques du médium. Par conséquent, il nous semble important d'exploiter cette possibilité pour attirer l'attention de l'utilisateur.

- des couleurs : il s'agit surtout du changement de couleur d'un ou plusieurs éléments afin de renseigner l'utilisateur sur le statut de son action.

- des éléments mobiles : des éléments à tirer (drag and drop) restent sur l'élément cible en cas de bonne réponse tandis que les éléments erronés retournent à leur place initiale.

\section{- Le retour pédagogique auditif :}

41 Comme nous avons recouru à des images symboliques, nous utilisons des sons à valeur symbolique pour marquer la réussite ou l'erreur.

\section{- Le retour pédagogique textuel :}

Fournir simplement un message écrit en tant que retour, ne nous a pas paru satisfaisant vu les possibilités offertes par le multimédia. C'est pourquoi nous avons misé sur une combinaison textuel - visuel ou textuel - auditif.

Texte avec soutien visuel : la couleur du texte indique si le retour est négatif (rouge) ou positif (vert). Notons que l'animation du texte représente une autre possibilité de soutien visuel.

Texte avec soutien auditif: les messages en pop-up, toujours accompagnés d'un son caractéristique, représentent l'exemple classique.

Paroles : un texte court enregistré (quelques mots) peut assurer les mêmes fonctions qu'un texte écrit et réagir à l'action de l'apprenant de façon informative, motivante ou même communicative (cf. supra). 
râce à des combinaisons entre types de feedback et modes de présentation (image avec ou sans animation, texte et son), on peut atteindre une assez grande variation de types de retour pédagogique - on notera que les variations que nous venons de présenter ne représentent que quelques exemples. Cela permet premièrement d'activer différents canaux de perception de l'apprenant pour focaliser son attention sur l'information, maintenir son intérêt tout en sollicitant le niveau affectif. C'est aux concepteurs que revient le choix réfléchi et parfois difficile du type de retour et de la modalité qui conviennent le mieux à chaque activité.

La vie du concepteur serait largement simplifiée si chacun de ses choix pédagogiques pouvait aisément et directement être mis en pratique. Malheureusement, il reste toujours confronté aux limites techniques qui, malgré toute l'imagination dont concepteurs et techniciens peuvent faire preuve, restent encore bien présentes. Et nous n'évoquerons pas les limites budgétaires qui obligent à prendre des décisions parfois douloureuses pour le didacticien. Nous pensons cependant avoir montré qu'il est possible de fournir des retours pédagogiques et des aides qui permettent de réaliser avec succès les tâches proposées, de corriger d'éventuelles erreurs et de les comprendre. Nous voyons là un début de réponse à l'interrogation de Maguy Pothier (et al.) qui se demande « comment concilier le fait que l'erreur permette d'apprendre [...] et la légitime frustration engendrée par l'erreur » (2000: 150). De plus, la simple mise à disposition de l'aide en complément de retours pédagogiques efficaces sans qu'elle ne soit jamais imposée permet de tenir compte de multiples stratégies d'apprentissage sans chercher à en privilégier une plus que l'autre. Cet aspect nous semble d'autant plus important que plusieurs chercheurs, dont Maguy Pothier et ses collègues, ont largement montré dans leurs résultats d'expérimentation que « des stratégies en tout point opposées peuvent aboutir à des résultats similaires" et que donc "faire travailler des gens à l'encontre de leurs stratégies "naturelles" n['est] guère rentable » (2000: 149).

\section{BIBLIOGRAPHIE}

Abé, Danièle \& M.J. Gremmo. 1985. « Teaching learning: redefining the teacher's role ». In Riley, Philip (dir.), Discourse and Learning. London : Longman, 233-247.

Anderson, John Robert. 2000. Cognitive psychology and its implications. New York : W. H. Freeman. Bélisle, Claire. 1998. « Enjeux et limites du multimédia en formation et en éducation ». Les Cahiers de l'Asdifle 7, 5-20.

Chamot, Anna Uhl \& J.M. O'Malley. 1994. The CALLA handbook: How to implement the Cognitive Academic Language Learning Approach. Reading, MA : Addison-Wesley.

Collectif de Chasseneuil. 2000. «Formations ouvertes et à distance : l'accompagnement pédagogique et organisationnel ». Conférence de Consensus 27, 28 \& 29 mars 2000. <http:// ressources.algora.org/reperes/tel/ccfod.pdf > Dernière consultation : septembre 2003.

Demaizière, Françoise \& C. Dubuisson. 1992. De l'EAO aux NTF - Utiliser l'ordinateur pour la formation. Paris : Ophrys. 
Demaizière, Françoise \& A.-L. Foucher. 1998. « Individualisation et initiative de l'apprenant dans des environnements d'apprentissage ouverts : une expérience d'autoformation guidée ». Études de Linguistique Appliquée 110, 226-237.

Dempsey, John V. \& W. Wager. 1988. « A Taxonomy for the timing of feedback in computer-based instruction ». Educational Technology 28/10, 20-25.

Duda, Richard. 1984. « Apprendre à apprendre les langues : 'Mais je veux être un handicapé linguistique'«. Mélanges Pédagogiques 15. Université de Nancy 2 : CRAPEL, 7-16.

Holec, Henri (dir.). 1985. Autonomy and self-directed learning: present fields of application. Council of Europe.

Hoska, Dorthy M. 1993. « Motivating learners through CBI feedback. Developing a positive learner perspective ». In Dempsey, John V. \& G. C. Sales (dir.), Interactive Instruction and Feedback. Englewood Cliffs : Educational Technology Publication, 105-132.

Keller, John. 2000. « How to integrate learner motivation planning into lesson planning: The ARCS model approach ». <http://www.netg.com/research/whitepapers/kellerwp.asp> Dernière consultation : décembre 2003.

Kulhavy, Raymond W. 1977. « Feedback in written instruction ». Review of Educational Research 47, 211-232.

McLaughlin, B., T. Rossman \& B. McLeod. 1983. « Second language learning: An information processing perspective ». Language Learning 33, 135-158.

Morin, Philippe. 2000. «La FOAD, de quoi s'agit-il ? De la définition au marché ». Forum "Formation à distance et technologies de la communication ", Actes du 14 décembre 2000, 28-35. <http:// www.cirffi.org/technologies/telechargement/Forum_foad_12-2000.pdf> Dernière consultation : septembre 2003.

Portine, Henri. 1998. «L"autonomie' de l'apprenant en questions ». ALSIC 1/1, 73-77. <http:// alsic.org> Dernière consultation : septembre 2003.

Pothier, Maguy, Anne Iotz \& Christine Rodrigues. 2000. « Les outils multimédia d'aide à 1 'apprentissage des langues : de l'évaluation à la réflexion prospective ». ALSIC $1 / 3,137-153$. < http://alsic.org> Dernière consultation : septembre 2003.

Rézeau, Joseph. 2001. « Médiatisation et médiation pédagogique dans un environnement multimédia. Le cas de l'apprentissage de l'anglais en Histoire de l'art à l'université ». Thèse pour le doctorat de l'Université Bordeaux 2. <http://joseph.rezeau.free.fr/theseNet/index.htm> Dernière consultation : septembre 2003.

Ross, Steven M. \& G. R. Morrison. 1993. « Using feedback to adapt instruction for individuals ». In Dempsey, John V. \& G. C. Sales (dir.), Interactive Instruction and Feedback. Englewood Cliffs : Educational Technology Publication, 177-195.

Sales, Gregory C. 1993. « Adapted and adaptive feedback in technology-based instruction ». In Dempsey John V. \& G. Sales (dir.), Interactive Instruction and Feedback. Englewood Cliffs : Educational Technology Publication, 159-175. 


\section{NOTES}

1. Le Centre de langues de l'Université de Salzburg a notamment coordonné le projet Babelnet et surtout Canal Rêve, la section de ce projet consacrée à l'apprentissage du français langue étrangère : <http://babelnet.sbg.ac.at/canalreve>.

2. Nous renvoyons, entre autres, aux travaux publiés par le Conseil de l'Europe (Holec 1985), aux nombreux articles publiés dans les Mélanges pédagogiques du CRAPEL, au numéro 41 (1981) d' Études de Linguistique Appliquée (Autonomie de l'apprentissage et apprentissage de l'autonomie) et aux autres publications plus récentes citées dans le corps du présent article.

3. Là encore, on se reportera au nombreux articles publiés dans les Mélanges pédagogiques du CRAPEL.

4. Retour pédagogique et feedback sont utilisés par la suite comme synonymes.

5. Cette conception se fonde en partie sur la définition de l'interactivité donnée par Claire Bélisle : « dispositif capable de réponses différenciées, en réaction à une intervention humaine ». (1998: 6)

\section{RÉSUMÉS}

En se fondant sur la notion d'autonomie, notion centrale en matière d'apprentissage sur des sites Internet, cet article fera ressortir l'importance d'accompagner et de guider l'apprenant dans sa prise d'autonomie et la construction de son projet et de ses parcours d'apprentissage. Il présentera notamment plusieurs possibilités d'aider l'apprenant à fixer et atteindre ses objectifs et quelques critères de qualité qui, s'ils sont respectés, devraient permettre une meilleure prise de contrôle de son apprentissage par l'utilisateur du site en lui laissant toujours le choix de ses cheminements et de l'ampleur de l'aide qu'il entend solliciter du système. Sur la base de récentes recherches menées par des didacticiens et techniciens spécialisés dans la conception de didacticiels, nous distinguerons ensuite les différentes formes de retours pédagogiques pour faire ressortir leurs spécificités et leur complémentarité pour un apprentissage efficace.

The article aims first of all at redefining the notion of autonomy, which is to be considered as a central concept in the domain of net-based learning. Secondly, it stresses the importance of leading and guiding learners towards autonomy, i.e. supporting learners to create their own projects and to structure their own learning path. It will be demonstrated how it is possible to help learners to reach their objectives. This entails the definition of quality criteria which allow learners to control their learning process, leaving it up to them at the same time to define their individual learning route and to determine the amount of support they want to have. Taking into consideration recent research in the field of internet-didactics and technology, we will present different types of feedback in order to point out their relevance for efficient autonomous learning. 
INDEX

Keywords : autonomy, e-learning, guidance, learner tutoring, pedagogical feedback

Mots-clés : aide à l'apprentissage, apprentissage en ligne, autonomie, guidage, retour pédagogique

\section{AUTEURS}

\section{CHRISTIAN OLLIVIER}

Christian Ollivier est Professeur agrégé d'allemand, docteur ès lettres, directeur du Centre de langues et didacticien au Département d'Études romanes de l'Université de Salzburg. Il est également formateur de formateurs, coordinateur de plusieurs projets de conception d'instruments d'apprentissage en ligne du FLE (Babelnet, Horizon 2000 on line, Net-Horizon). Ses recherches et publications s'inscrivent dans le domaine de l'apprentissage en ligne des langues ( Perspectives fle, Le français dans le monde, Tell\&CALL, contact+, UNTELE...).

ollivier.christian@gmail.com

\section{GERDA WEISS}

Gerda Weiss est Maître ès lettres ; elle a écrit un mémoire sur la gestion du savoir dans les classes équipées d'ordinateurs portables. Elle collabore à plusieurs projets de conception d'instruments d'apprentissage en ligne du FLE (Babelnet, Horizon 2000 on line, Net-Horizon). Elle a participé à la conception et à la réalisation de matériels d'apprentissage en ligne du FLE. Elle prépare une thèse sur l'apprentissage autonome en ligne.gerda_we@hotmail.com 